\title{
Motivos de participación deportiva y satisfacción intrínseca en jugadores de pádel Motives for sports participation and intrinsic satisfaction in padel players
}

Alberto Rodríguez Cayetano, Salvador Pérez Muñoz, José Manuel de Mena Ramos, Nuria Codón Beneitez, Antonio Sánchez Muñoz

Universidad Pontificia de Salamanca (España)

\begin{abstract}
Resumen. El objetivo principal de este estudio es el de analizar las razones para la realización de práctica deportiva y su nivel de satisfacción intrínseca en una muestra de 146 jugadores de pádel, 82 hombres y 64 mujeres con una media de edad de 39.46 ( \pm 11.60$)$ años y que entrenan, al menos, dos horas semanales. Para ello, se han utilizado dos cuestionarios: el cuestionario de Motivos de Participación (PMQ) y el cuestionario de Satisfacción Intrínseca en el Deporte (SSI). Los resultados obtenidos muestran que el factor más destacado para la práctica deportiva en los jugadores de pádel es el de amistad / grupo de iguales mientras que el factor menos valorado es el del estatus social. Además, el placer interno de los jugadores se basa en la diversión por la práctica del pádel y no en el aburrimiento. Por lo tanto, los clubes deben tener en cuenta la realización de tareas sociales y lúdicas para mantener y aumentar el número de practicantes en esta modalidad deportiva.
\end{abstract}

Palabras Clave: Psicología Deportiva; Pádel; Motivación Intrínseca; Motivación Extrínseca; Satisfacción.

Abstract. The main aim of this study was to analyze the reasons for practicing sports and the level of intrinsic satisfaction in a sample of 146 paddle players (82 men and 64 women) with average age of $39.46( \pm 11.60)$ years old who trained at least two hours per week. For this, two questionnaires were used: the Participation Motivation Questionnaire (PMQ), and the Intrinsic Satisfaction in Sport Questionnaire. The results show that the factor that stands out as a reason to practice sport in paddle players is friendship / group of peers, whilst the lowest valued factor is social status. In addition, internal pleasure of the players is based on fun of playing paddle and not on boredom. Therefore, clubs must take into account and propose social and recreational tasks in order to maintain and increase the number of practitioners of this sport.

Keywords: Sports Psychology; Paddle; Intrinsic Motivation; Extrinsic Motivation; Satisfaction.

\section{Introducción}

El pádel es uno de los deportes más practicados en España en la actualidad teniendo un gran auge en los últimos años, tanto a nivel de número de practicantes como en el crecimiento del número de licencias (Consejo Superior de Deportes, 2015). Este auge ha producido un aumento en el número de investigaciones en torno a esta modalidad deportiva (Courel-Ibáñez, Sánchez-Alcaraz, García \& Echegaray, 2017). El pádel se caracteriza por esfuerzos intermitentes, combinando puntos de larga duración y baja intensidad $(9$ a 15 segundos de media) con una alta participación de los jugadores (Courel-Ibáñez, Sánchez-Alcaraz \& Cañas, 2017; Courel-Ibañez, Sánchez-Alcaraz \& Muñoz, 2019; GarcíaBenítez, Courel-Ibáñez, Pérez-Bilbao \& Felipe, 2018)

El deporte del pádel se constituye como un deporte relativamente joven en el ámbito del alto rendimiento deportivo (Sánchez-Alcaraz, 2013). Pese a su juventud, en los últimos años se ha multiplicado el interés científico para conocer las exigencias técnico-tácticas, físicas, biomecánicas y psicológicas específicas de este deporte (Courel-Ibáñez \& SánchezAlcaraz, 2018; Courel-Ibáñez, Sánchez-Alcaraz \& Sánchez, Alcaraz, 2017; Lupo et al., 2018; Muñoz, et al., 2016; Rodríguez-Cayetano et al., 2017; Sánchez-Alcaraz, CourelIbáñez \& Cañas, 2018; Sañudo, De Hoyo \& Carrasco, 2008). Desde una perspectiva psicológica, aspectos como el control de la activación, la mejora del estado de ánimo, la concentración y la visualización suponen algunos de los requerimientos a nivel psicológico, debiéndose disponer de información determinada no sólo con componentes estrictamen-

Fecha recepción: 30-09-19. Fecha de aceptación: 09-01-20 Alberto Rodríguez Cayetano

arodriguezca@upsa.es te relacionados con el ámbito deportivo, sino también con el funcionamiento psicológico global del deportista en su entorno (Ruiz \& Lorenzo, 2008). Díaz et al. (2018) demostraron como el estado de ánimo mejoró en todos sus factores (tensión, depresión, angustia, vigor, fatiga y cansancio) gracias a la práctica de pádel en una muestra de mujeres adultas.

Por su parte, la motivación es un elemento clave para lograr el compromiso y la adherencia al deporte, ya que es el más importante e inmediato determinante del comportamiento humano, pues lo despierta, le da energía, lo dirige y lo regula, siendo por tanto un mecanismo psicológico que gobierna la dirección, intensidad y persistencia de la conducta (Moreno, Cervelló \& González, 2007). El estudio de la motivación hacia la actividad física y el deporte ha sido un aspecto que históricamente ha preocupado, entre otros, a los entrenadores, profesores de Educación Física, psicólogos y preparadores físicos (Dosil, 2004; Martínez et al., 2008; Moreno-Murcia et al., 2014).

Las motivaciones son conceptos dinámicos, ya que varían en función del tiempo de práctica del jugador. En el inicio, aparecen motivaciones dispersas. Durante el mantenimiento, la motivación se centra en la competición, en la tarea y en el esfuerzo. En la etapa de cambio deportivo, se buscan nuevas sensaciones o conseguir objetivos hasta ahora no logrados, mientras que el abandono se produce cuando las motivaciones están centradas en la eliminación de aspectos negativos (Moreno \& Cervelló, 2010).

En una sociedad donde hay más tiempo de ocio la práctica de actividades físico-deportivas ocupa un papel importante, y los estudios realizados muestran un progreso en cuanto al número de individuos implicados en la participación en actividades físico-deportivas, quedando aún un largo camino para establecer la actividad física como un hábito de práctica entre la población. Conocer los factores que 
incentivan en las personas el desarrollo de una determinada actividad deportiva, puede servir para crear programas de entrenamiento que respeten los intereses de aquellos que lo practican (González, Tabernero \& Márquez, 2000). Este hecho es fundamental ya que, estudios recientes, relacionan la práctica de pádel con mejores niveles de condición física y, por consiguiente, la mejora de la salud en mujeres adultas de mediana edad (Courel-Ibáñez et al., 2018).

La satisfacción generada por una práctica social, una actividad o la participación en un programa, constituye un indicador del comportamiento y de la implicación de las personas en dicha actividad. A mayor satisfacción, más motivación y mejor logro de los objetivos que promueve el programa. El interés por éste ámbito de estudio radica en la potencialidad de la satisfacción personal como factor que promueve una disposición favorable en un ámbito de acción determinado y mayor facilidad para alcanzar los objetivos que se promueven en dicho ámbito (Chen, 2001).

En ese marco de referencia, la satisfacción con el pádel constituye un indicador que refleja la disposición con la que los alumnos y las alumnas acuden a las clases de pádel. Esta satisfacción condicionará la motivación y, por tanto, la implicación personal en el logro de los objetivos del aprendizaje (Hernández, Velázquez \& Aguado, 2010). En este sentido, son varios los estudios que han analizado los motivos de la práctica de pádel en adultos. Sánchez-Alcaraz et al. (2018) mostraron que, para una muestra de 77 jugadores, el factor más valorado para la práctica de este deporte es el disfrute, mientras que el menos valorado es el de la apariencia, sin que existan diferencias significativas entre ambos sexos. Estos resultados van en concordancia al estudio realizado por Courel-Ibáñez et al. (2018) en el que el disfrute es el factor más valorado por ambos sexos de cualquier edad, experiencia o nivel de juego.

El objetivo principal de esta investigación es el de analizar cuáles son los motivos de participación deportiva y la satisfacción intrínseca en jugadores de pádel durante el año 2018 en función del sexo, de la edad y del lado en el que juegan habitualmente.

\section{Material y método}

\section{Muestra}

Este estudio se realizó entre jugadores y jugadoras de pádel de España durante el año 2018. Fueron partícipes de este estudio un total de 146 sujetos, 82 jugadores y 64 jugadoras, de entre 16 y 60 años y que entrenan, al menos, 2 horas semanales. La media de edad de los participantes es de $39.46( \pm 11.60)$. Según el lado en el que suelen jugar más cómodo, 79 de ellos suelen jugar en el lado derecho y 67 en el lado izquierdo.

\section{Instrumentos}

Motivos de participación deportiva: se administró el Cuestionario de Motivos de Participación Deportiva (PMQ) de Gill, Gross y Huddleston (1983) en su versión española utilizada por López y Márquez (2001). Este cuestionario consta de 35 ítems, cada uno de los cuales supone un posible motivo de práctica deportiva, a los que se responde mediante una escala tipo Likert de cinco valores (desde uno, «nada importante» a cinco, «muy importante». Estos ítems se clasifican en ocho factores: Estatus Social, Salud/Forma Física, Diversión/Socialización, Cooperación/Trabajo en Equipo, Competición, Liberar Energía/Catarsis, Amistad/Grupo de Iguales y Autosuperación. La validez factorial del cuestionario para la participación deportiva fue investigada por Durand (1988), estableciendo la consistencia interna con un alfa de Cronbach de 0.84 .

Satisfacción intrínseca: se utilizó el Cuestionario de Satisfacción Intrínseca en el Deporte (SSI) de Duda y Nicholls (1992) en su versión en castellano realizada por Balaguer, Atienza, Castillo, Moreno y Duda (1997). El cuestionario consta de ocho ítems divididos en dos escalas que miden la Diversión (5 ítems) y el Aburrimiento (3 ítems). Se valoran las respuestas con una escala tipo Likert que oscila entre uno «muy en desacuerdo» a cinco «muy de acuerdo». Diversos estudios han medida la validez del cuestionario (Castillo, Balaguer \& Duda, 2002; Castillo, Balaguer, Duda \& GarcíaMerita, 2004; Ruiz-Juan, Gómez, Pappous, Alacid \& Flores, 2010; Zarauz \& Ruiz-Juan, 2014) con valores en el alfa de Cronbach que oscilan entre el 0.75 al 0.87 en el factor de Satisfacción/Diversión y entre 0.71 a 0.78 en el factor Aburrimiento.

\section{Procedimiento}

Para la administración de los cuestionarios, se solicitó la colaboración de clubes nacionales de pádel para obtener su visto bueno. Antes de la realización de los cuestionarios, los sujetos fueron informados del objetivo del estudio, voluntariedad y absoluta confidencialidad de las respuestas y el manejo de los datos cumpliéndose la Declaración Ética de Helsinki. En el caso de los jugadores menores de edad, se solicitó, además, un consentimiento informado de los padres.

La toma de datos tuvo lugar dentro de las instalaciones de los diferentes clubes, siempre en presencia de los investigadores, para resolver todas las cuestiones que se produjeran.

\section{Análisis de datos}

Para el análisis de los datos, se calcularon los descriptivos principales (Media y desviación típica). Posteriormente, se realizó un análisis univariante (ANOVA) para estudiar si había diferencias significativas entre los factores que conformaban el cuestionario y en el caso de observar diferencias significativas, se utilizó la prueba de comparaciones múltiples Scheffé para detectar los factores concretos entre los que se producían tales diferencias. Las diferencias entre los resultados se consideran significativas si $p<.05$. Por último, se analizaron las correlaciones para determinar las relaciones entre los factores. Para todo ello, se ha utilizado el paquete estadístico SPSS para Windows v.20, (IBM SPSS Statistiscs for Windows, Version 20.0. Armonk, NY: IBMCorp).

\section{Resultados}

Resultados descriptivos motivos de participación deportiva (PMQ)

A continuación, se muestran los resultados descriptivos generales de los factores que conforman los motivos de par- 
ticipación deportiva. El mayor motivo por el que los jugadores de pádel escogen practicar este deporte es la amistad / grupo de iguales $(\mathrm{M}=4.53 \pm .64)$, seguido de la cooperación / trabajo en equipo $(M=4.25 \pm .77)$, sin embargo, el motivo al que menos importancia le dan es al estatus social $(\mathrm{M}=2.01$ $\pm .78)$ (Tabla 1).

En la tabla 2, se muestran los resultados descriptivos de los factores que conforman los motivos de participación deportiva en función del sexo. El mayor motivo de participación para los jugadores de sexo masculino es el de amistad / grupo de iguales $(\mathrm{M}=4.52 \pm .67)$, seguido del de cooperación / trabajo en equipo $(\mathrm{M}=4.06 \pm .82)$. En el caso de las jugadoras, el mayor motivo de participación coincide con los jugadores, aunque el factor cooperación/ trabajo en equipo es algo más elevado en el sexo femenino $(\mathrm{M}=4.48 \pm .62)$. El motivo al que menos importancia le da tanto el sexo masculino como el femenino es al estatus social $(\mathrm{M}=2.05 \pm .78)$ y $(\mathrm{M}=1.97 \pm .78)$. Por último, se refleja que existen diferencias significativas $(p<.05)$ en los factores de salud/forma física, cooperación/trabajo en equipo y liberar energía/catarsis.

Con respecto a la edad de los deportistas, en la tabla 3 se presentan los resultados descriptivos, diferenciando entre jugadores menores de 25 años, entre 26 y 45 años y mayores de 46 años. El mayor motivo para las tres franjas de edad es el de amistad /grupo de iguales con medias de 4.36 ( \pm .76$)$, $4.49( \pm .65)$ y $4.67( \pm .52)$, respectivamente. También coinciden en el motivo que menos le incita a la práctica del pádel, que es el estatus social, con medias de $1.92( \pm .81), 1.99( \pm .79)$ y $2.10( \pm .76)$. Para finalizar, se observan diferencias significativas $(p<.05)$ en los factores de salud/forma física y en el de diversión/socialización. La tabla 4 muestra que existen diferencias significativas en el factor salud/forma física entre los menores de 25 años y los jugadores de entre 26 y 45 años y en el factor diversión/socialización entre los menores de 25 años y los mayores de 46 años.

Por último, se señalan los resultados descriptivos de los factores que conforman los motivos de participación deportiva en función del lado en el que juegan. El factor más valorado tanto para los jugadores que juegan en el lado de la derecha como para los jugadores que juegan en el lado de revés es la amistad/grupo de iguales $(\mathrm{M}=4.57 \pm .59)$ y $(\mathrm{M}=$ $4.48 \pm .68)$, dándole una mayor importancia los jugadores que juegan en el lado de iguales. También coinciden en el motivo que menos le incita a la práctica del pádel, que es el estatus social, siendo menor aún para los jugadores que juegan en el lado derecho $(\mathrm{M}=1.95 \pm .85)$ que para los que juegan en el revés $(\mathrm{M}=2.09 \pm .69)$ (Tabla 5). Existen diferencias significativas $(p<.05)$ entre estos jugadores en los factores de Competición y Autosuperación.

\section{Resultados descriptivos en la satisfacción intrínseca}

\section{en el deporte (SSI)}

En la tabla 6, se muestran los resultados descriptivos generales de los factores que conforman la satisfacción intrínseca en el deporte. Los jugadores de pádel basan su satisfacción intrínseca en la diversión $(\mathrm{M}=4.53 \pm .53)$, y no en el aburrimiento $(\mathrm{M}=1.40 \pm .58)$.

Los jugadores masculinos basan su satisfacción intrínseca principalmente en la diversión $(\mathrm{M}=4.45 \pm .55)$ y no en el aburrimiento $(\mathrm{M}=1.39 \pm .54)$, esto se repite en el caso de las
Tabla 1.

Resultados descriptivos generales de los factores que conforman los motivos de participación deportiva de jugadores de pádel

COOP COMP LBE \begin{tabular}{ccccccccc}
\hline & EST SOC & S/FF & DIVE & COOP & COMP & LIBE & AMIS & AUTO \\
\hline $\mathrm{N}$ & 146 & 146 & 146 & 146 & 146 & 146 & 146 & 146 \\
. & 2.01 & 4.08 & 3.76 & 4.25 & 3.61 & 3.21 & 4.53 & 3.88 \\
DT & .78 & .60 & .67 & .77 & .95 & .74 & .64 & .69
\end{tabular}

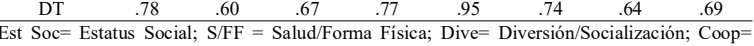
Est Soc $=$ Estatus Social; S/FF $=$ Salud $/$ Forma Física; Dive $=$ Diversion $/$ Socialización; Coop=
Cooperación/Trabajo en Equipo; Comp= Competición; Libe $=$ Liberar energí/Catarsis; Amis: Amistad/Grupo de Iguales; Auto= Autosuperación

Tabla 2

Resultados descriptivos de los factores que conforman los motivos de participación deportiva en función del sexo

\begin{tabular}{ccccccccc} 
& EST SOC & S/FF & DIVE & COOP & COMP & LIBE & AMIS & AUTO \\
\hline Masculino & 82 & 82 & 82 & 82 & 82 & 82 & 82 & 82 \\
. & 2.05 & 3.96 & 3.82 & 4.06 & 3.65 & 3.05 & 4.52 & 3.80 \\
DT & .78 & .55 & .63 & .82 & .94 & .70 & .67 & .64 \\
\hline Femenino & 64 & 64 & 64 & 64 & 64 & 64 & 64 & 64 \\
. & 1.97 & 4.22 & 3.68 & 4.48 & 3.56 & 3.41 & 4.53 & 3.98 \\
DT & .78 & .63 & .71 & .62 & .97 & .75 & .59 & .75 \\
\hline Sig. & .540 & $.010^{*}$ & .206 & $.001^{*}$ & .598 & $.004^{*}$ & .949 & .119 \\
\hline
\end{tabular}

Est Soc $=$ Estatus Social; $\mathrm{S} / \mathrm{FF}=$ Salud/Forma Física; Dive $=$ Diversión $/$ Socialización; Coop= Cooperación/Trabajo en Equipo; Comp= Competición; Libe= Liberar energia/Catarsis; Amis: Amistad/Grupo de Iguales; Auto= Autosuperación

${ }^{*} p<.05$

Tabla 3.

Resultados descriptivos de los factores que conforman los motivos de participación deportiva en función de la edad

\begin{tabular}{ccccccccc}
\hline & EST SOC & S/FF & DIVE & COOP & COMP & LIBE & AMIS & AUTO \\
\hline$<25$ años & 25 & 25 & 25 & 25 & 25 & 25 & 25 & 25 \\
. & 1.92 & 3.80 & 3.49 & 4.32 & 3.76 & 3.36 & 4.36 & 4.04 \\
DT & .81 & .58 & .65 & .69 & .83 & .86 & .76 & .74 \\
\hline $26-45$ años & 64 & 64 & 64 & 64 & 64 & 64 & 64 & 64 \\
. & 1.97 & 4.22 & 3.68 & 4.48 & 3.56 & 3.41 & 4.53 & 3.98 \\
DT & .78 & .63 & .71 & .62 & .97 & .75 & .59 & .75 \\
\hline$>46$ años & 51 & 51 & 51 & 51 & 51 & 51 & 51 & 51 \\
. & 2.10 & 4.10 & 3.92 & 4.12 & 3.35 & 3.10 & 4.67 & 3.75 \\
DT & .76 & .50 & .63 & .82 & .98 & .64 & .52 & .69 \\
\hline Sig. & .595 & $.035^{*}$ & $.026^{*}$ & .332 & .056 & .332 & .105 & .163 \\
\hline
\end{tabular}

Est Soc= Estatus Social; S/FF = Salud/Forma Física; Dive= Diversión/Socialización; Coop= Cooperación/Trabajo en Equipo; Comp= Competición; Libe= Liberar energia/Catarsis; Amis: Amistad/Grupo de Iguales; Auto= Autosuperación

${ }^{*} p<.05$

Tabla 4

Comparaciones múltiples Scheffé del ANOVA de los factores Salud / Forma Física y Diversión , Socialización en función de la edad de los jugadores

\begin{tabular}{|c|c|c|c|c|c|}
\hline Variable dependiente & $\begin{array}{l}\text { (I) Edad del } \\
\text { Deportista }\end{array}$ & $\begin{array}{l}\text { (J) Edad del } \\
\text { Deportista }\end{array}$ & $\begin{array}{c}\text { Diferencia de } \\
\text { medias (I-J) }\end{array}$ & $\begin{array}{l}\text { Error } \\
\text { típico }\end{array}$ & Si \\
\hline \multirow{6}{*}{$\begin{array}{l}\text { Salud/ } \\
\text { Forma física }\end{array}$} & \multirow{2}{*}{ Menos de 25 años } & Entre 26 y 45 años & $-.357^{*}$ & .137 & .03 \\
\hline & & Mayores de 46 años & -.298 & .144 & 12 \\
\hline & \multirow{2}{*}{ Entre 26 y 45 años } & Menos de 25 años & $.357^{*}$ & .137 & \\
\hline & & Mayores de 46 años & .059 & .109 & \\
\hline & \multirow{2}{*}{ Mayores de 46 años } & Menos de 25 años & 298 & .144 & \\
\hline & & Entre 26 y 45 años & -.059 & .109 & \\
\hline \multirow{6}{*}{$\begin{array}{l}\text { Diversión/ } \\
\text { Socialización }\end{array}$} & \multirow{2}{*}{ Menos de 25 años } & Entre 26 y 45 años & -239 & .153 & \\
\hline & & Mayores de 46 años & $-.432^{*}$ & .160 & \\
\hline & \multirow{2}{*}{ Entre 26 y 45 años } & Menos de 25 años & .239 & .153 & \\
\hline & & Mayores de 46 años & -193 & .121 & \\
\hline & \multirow{2}{*}{ Mayores de 46 años } & Menos de 25 años & $.432^{*}$ & .160 & .02 \\
\hline & & Entre 26 y 45 años & 193 & .121 & .2 \\
\hline
\end{tabular}

${ }^{*} p<.05$

Tabla 5 .

Resultados descriptivos de los factores que conforman los motivos de participación deportiva en

\begin{tabular}{cccccccc} 
función del lado en el que juegan & & & & & & \\
\hline EST SOC & S/FF & DIVE & COOP & COMP & LIBE & AMIS & AUTO
\end{tabular}

\begin{tabular}{|c|c|c|c|c|c|c|c|c|}
\hline & EST SOC & & DIVE & & & & & \\
\hline Derecha & 79 & 79 & 79 & 79 & 79 & 79 & 79 & 79 \\
\hline & 1.95 & 4.06 & 3.76 & 4.19 & 3.43 & 3.18 & 4.57 & 3.76 \\
\hline DT & .85 & .61 & .70 & .79 & .99 & .76 & .59 & .74 \\
\hline Izquierda & 67 & 67 & 67 & 67 & 67 & 67 & 67 & 67 \\
\hline & 2.09 & 4.09 & 3.75 & 4.31 & 3.82 & 3.24 & 4.48 & 4.03 \\
\hline DT & .69 & .60 & .64 & .74 & .85 & .72 & .68 & .60 \\
\hline Sig. & .280 & .793 & .883 & .333 & $.013^{*}$ & .619 & .385 & $.018^{*}$ \\
\hline $\begin{array}{l}\text { Est Soc }=\mathrm{E} \\
\text { Cooperación } \\
\text { Amistad/Gr } \\
{ }^{*} p<.05\end{array}$ & $\begin{array}{l}\text { atus Socia } \\
\text { Trabajo en } \\
\text { o de Igual }\end{array}$ & $\begin{array}{l}\text { S/FF } \\
\text { Equipo } \\
\text {; Auto }\end{array}$ & $\begin{array}{l}\text { Salud/Fo } \\
\text { Comp= } \\
\text { Autosupe }\end{array}$ & $\begin{array}{l}\text { na Físic } \\
\text { mpetició } \\
\text { ción }\end{array}$ & $\begin{array}{l}\text { Dive }= \\
\text { Libe }=\end{array}$ & $\begin{array}{l}\text { iversión } \\
\text { iberar e }\end{array}$ & $\begin{array}{l}\text { ocializac } \\
\text { rgia/Cate }\end{array}$ & $\begin{array}{l}\text {;; Coop= } \\
\text { is; Amis }\end{array}$ \\
\hline $\begin{array}{l}\text { Tabla } 6 . \\
\text { Resultados } \\
\text { deporte }\end{array}$ & criptivos & nerale & de los fac & ores que & onform & & ción $i$ & ieca en e \\
\hline & & & & DIVER & IÓN & & SURRIM & NTO \\
\hline & $\mathrm{N}$ & & & $\begin{array}{l}14 \\
4.5\end{array}$ & & & $\begin{array}{l}146 \\
1.40\end{array}$ & \\
\hline & DT & & & .5 & & & .58 & \\
\hline
\end{tabular}

Tabla 7.

Resultados descriptivos generales de los factores que conforman la satisfacción intrínseca en el \begin{tabular}{ccc} 
deporte en función del sexo, la edad y el lado en el que juegan & \\
\hline $\mathrm{N}$ & DIVERSIÓN & ABURRIMIENTO \\
\hline
\end{tabular}

\begin{tabular}{cccc}
\hline & $\mathrm{N}$ & DIVERSIÓN & ABURRIMIENTO \\
\hline Masculino & 82 & $4.45( \pm .55)$ & $1.39( \pm 1.41)$ \\
Femenino & 64 & $4.63( \pm .49)$ & $1.41( \pm .64)$ \\
Sig. & & $.048^{*}$ & .869 \\
\hline$<25$ años & 25 & $4.52( \pm .51)$ & $1.48( \pm .51)$ \\
$26-45$ años & 70 & $4.56( \pm .53)$ & $1.36( \pm .64)$ \\
$>46$ años & 51 & $4.49( \pm .54)$ & $1.41( \pm .54)$ \\
Sig. & & .789 & .650 \\
\hline Derecha & 79 & $4.54( \pm .01)$ & $1.34( \pm .50)$ \\
Izquierda & 67 & $4.51( \pm .56)$ & $1.46( \pm .66)$ \\
Sig. & & .676 & .211 \\
\hline${ }^{*} p<.05$ & & &
\end{tabular}


jugadoras femeninas, que obtienen un resultado de media de $4.63( \pm .49)$ para la diversión, por lo que le dan más importancia que los jugadores masculinos, existiendo diferencias significativas entre ambos sexos, y de $1.41( \pm .64)$ para el aburrimiento.

En función de la edad de los deportistas, las tres franjas de edades basan su satisfacción intrínseca en la satisfacción/diversión, con medias de $4.52( \pm .51), 4.55( \pm .53)$ y 4.49 $( \pm .54)$, respectivamente, y no en el aburrimiento, $1.48( \pm .51)$, $1.36( \pm .64)$ y $1.41( \pm .54)$. Por lo tanto, los que más se apoyan en la diversión son los jugadores que tienen edades comprendidas entre los 26 y 45 años.

Por último, tanto los jugadores que juegan en la derecha como los jugadores que juegan en el revés basan su satisfacción intrínseca en la diversión con una media de 4.54 ( \pm $.50)$ y $4.51( \pm .56)$, respectivamente, y no en el aburrimiento $1.34( \pm .50)$ y $1.46( \pm .66)$ (Tabla 7$)$.

Correlaciones entre los factores que conforman los motivos de participación deportiva y la satisfacción intrínseca en el deporte

A continuación, se muestran los coeficientes de correlación de Pearson entre los factores que conforman los motivos de participación deportiva y la satisfacción intrínseca en el deporte (Tabla 8).

- Respecto al factor estatus social existe correlación positiva significativa con la diversión/socialización, competición, liberar energía/catarsis y autosuperación y correlación positiva significante con la amistad/grupo de iguales.

- Siguiendo con el factor salud/forma física existe correlación positiva significativa con la competición, liberar energía/catarsis, amistad/grupo de iguales y autosuperación y correlación positiva significante con la diversión/socialización y la cooperación/trabajo en equipo.

- En relación al factor diversión/socialización existe correlación positiva significativa con la cooperación/trabajo en equipo, liberar energía/catarsis, amistad/grupo de iguales y autosuperación.

- Respecto a la cooperación/trabajo en equipo existe correlación positiva significativa con la competición, liberar energía/catarsis, amistad/grupo de iguales y autosuperación.

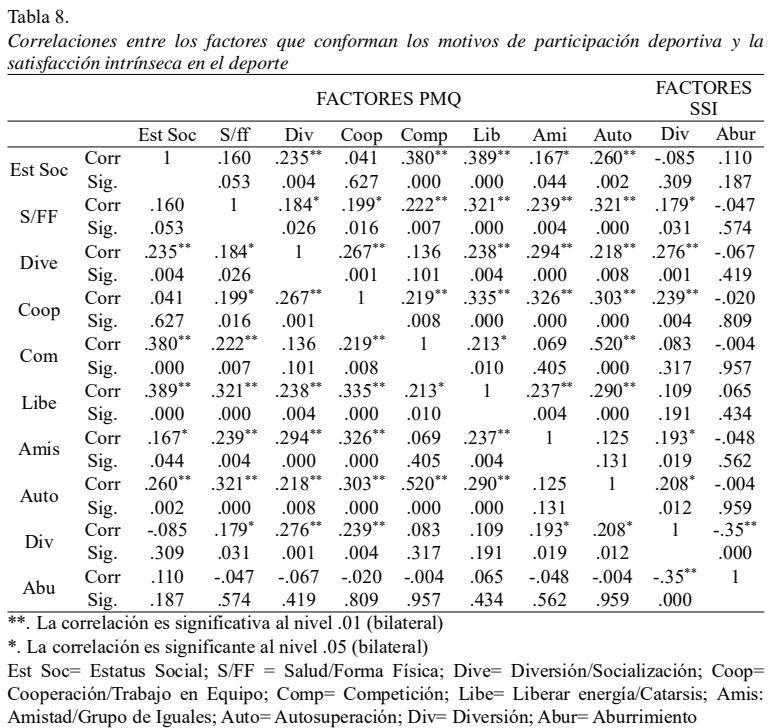

- Por su parte, el factor competición mantiene correlación positiva significante con liberar energía/catarsis y correlación positiva significativa con la autosuperación.

- En penúltimo lugar, en el factor liberar energía/catarsis existe correlación positiva significativa con la amistad/ grupo de iguales y la autosuperación.

- Por último, se observa que existe una correlación negativa significativa entre la satisfacción/diversión y el aburrimiento, sin embargo, hay una correlación positiva significante entre la satisfacción/diversión y la salud/forma física, amistad/grupo de iguales y autosuperación y correlación positiva significativa entre la satisfacción/diversión y la diversión/socialización y cooperación/trabajo en equipo.

\section{Discusión}

El objetivo de estudio fue analizar los motivos de participación en jugadores de pádel y examinar las diferencias en función del sexo, la edad y el lado en el que jugaban. Los resultados generales mostraron que el factor principal para la práctica de esta modalidad deportiva es el de la amistad/ grupo de iguales, seguido por la cooperación/trabajo en equipo y de la salud/forma física, mientras que el motivo que menos trascendencia tuvo fue el del estatus social. Estudios realizados en otros deportes, González, Tabernero y Márquez (2000) con jugadores de fútbol y tenis, Martínez et al., (2012) con adolescentes españoles y Zubiaur y Del Riego (2015) con practicantes de BMX, mostraron que los factores ligados a la diversión, la realización de ejercicio físico o estar con amigos, se encuentran entre los más valorados por estos deportistas.

Con respecto a la satisfacción intrínseca de los jugadores de pádel, se estableció una correlación negativa significativa entre la diversión y el aburrimiento, siendo este primer factor, el más valorado por los jugadores. Estos resultados siguen la línea de Courel- Ibáñez et al. (2018) y SánchezAlcaraz et al. (2018) con jugadores de pádel, en el que mostraron un predominio de la diversión como principal factor de motivación hacia la práctica deportiva y el de apariencia como el menos valorado. Estos datos pueden deberse al carácter social y de disfrute que tiene el pádel (Courel-Ibáñez et al., 2016; Lasaga, 2011). Por lo tanto, para mantener o aumentar la adherencia de los jugadores hacia esta actividad deportiva, se debe de tener en cuenta el disfrute y el aspecto social de este deporte, a la hora de proponer actividades para fidelizar a estos deportistas y promover la aparición de hábitos de vida saludables (Carron, Hausenblas \& Estabrooks, 2003; Garita, 2006).

En cuanto a los resultados obtenidos en función del sexo de los jugadores, el mayor motivo de participación en el pádel para los jugadores de sexo masculino es el de amistad / grupo de iguales, seguido del de cooperación / trabajo en equipo; en el caso de las jugadoras, los mayores motivos de participación coinciden con éstos, aunque el factor de factor cooperación / trabajo en equipo es más elevado. Estos resultados van en la línea del estudio realización por Pavón y Moreno (2008) con universitarios, en el que las chicas muestran una mayor preferencia por las actividades de carácter más cooperativo y menos individualista y los intereses de los chicos se centran en carácter más activo y competitivo. 
Estos últimos datos, no coinciden con otras investigaciones similares en las que los hombres valoraban más que las mujeres el aspecto social y la mejora de la salud en la realización de práctica deportiva (Courel-Ibáñez et al., 2018; García-Puello, Herazo \& Tuescas, 2015; Sánchez-Alcaraz et al., 2018). Es por ello que, el pádel puede ser un buen aliciente para promover una práctica deportiva saludable entre la población adulta. Por su parte, el motivo al que menos importancia da, tanto el sexo masculino como el femenino es al estatus social, siendo menor en ellas, al igual que en el estudio realizado por Sánchez-Alcaraz, (2018) en el que el factor apariencia fue el menos valorado.

Con respecto a la edad de los participantes, el factor al que mayor importancia le dan es el de amistad / grupo de iguales. También coinciden en el motivo que menos le incita a la práctica del pádel, que es el estatus social, si bien, va aumentando la importancia de este factor con el paso de los años. Estos resultados van en la línea del estudio realizado por Pérez, Sanchez y Urchaga (2015) con jugadores de fútbol, en el que se pone de manifiesto que los motivos de participación «Intrínseca-Deporte» y «Logro», están presentes en todas las categorías y edades, sin mostrar diferencias significativas en función de la edad. Por el contrario, en la investigación realizada por Nuviala, Gómez, Grao, Granero y Nuviala (2013) demostraron que el factor salud/forma física iba en aumento con el paso de los años, mientras que en nuestro estudio el grupo de jugadores entre 25 y los 45 años son los que más valoran este factor.

Por último, en función del lado en que juegan de forma habitual, los resultados reflejan que el mayor motivo tanto para los jugadores que juegan en el lado derecho como para los jugadores que juegan en el revés es la amistad / grupo de iguales. También coinciden en el motivo que menos les incita a la práctica del pádel que es el estatus social. Cabe destacar, que los jugadores que juegan en el lado izquierdo, los factores de la autosuperación y la competición, son mayores que los que juegan en el lado derecho. Esto puede ser debido a que los deportistas que juegan en el lado de la ventaja, suelen ser jugadores con una mayor potencia y con un mayor carácter competitivo, ya que son los que comienzan el punto en los momentos clave del partido y deben de soportar esa responsabilidad.

Este estudio presenta algunas limitaciones que deben ser tenidas en cuenta a la hora de interpretar los resultados. En primer lugar, debido al reducido tamaño muestral, no se han podido establecer comparaciones más exhaustivas entre las variables estudiadas. La información obtenida podría ser de gran interés para estudiar las diferencias entre deportistas profesionales de pádel y deportistas amateurs, así como comparar los motivos de participación deportiva con jugadores federados de tenis, intentando buscar una explicación al decrecimiento de número de licencias en tenis y el aumento en el pádel. Igualmente, sería interesante comparar los motivos de participación deportiva con el rendimiento obtenido por esos jugadores federados a lo largo de una temporada, para analizar si influyen en el rendimiento de los mismos.

\section{Conclusiones}

En conclusión, el estudio realizado muestra que los moti- vos de participación de los jugadores de pádel encuestados basan su práctica deportiva en compartir experiencias con sus amigos, así como practicar una actividad física saludable y el trabajo en equipo.

Estas afirmaciones, anteriormente descritas, son muy importantes para que en los clubes de pádel, tanto los gestores como los directores deportivos, implanten actividades organizadas bajo esta premisa para fidelizar a sus jugadores en la práctica deportiva, ya que en muchas ocasiones se basan demasiado en tareas competitivas, no siendo este factor uno de los más importantes para estos deportistas.

\section{Referencias}

Balaguer, I., Atienza, F.L., Castillo, I., Moreno, Y. \& Duda, J.L. (1997). Factorial structure of measures of satisfaction/ interest in sport and classroom in the case of Spanish adolescents. Fourth European Conference of Psychological Assesment. Lisboa, Portugal.

Carron, A. V., Hausenblas, H. A. \& Estabrooks, P. A. (2003). The psychology of physical activity (vol. 1). McGrawHill Companies.

Castillo, I., Balaguer, I. \& Duda, J.L. (2002). Las perspectivas de meta de los adolescentes en el contexto deportivo. Psicothema, 14(2), 280-287.

Castillo, I., Balaguer, I., Duda, J.L. \& García-Merita, M. L. (2004). Factores psicosociales asociados con la participación deportiva en la adolescencia. Revista Latinoamericana de Psicología, 36(3), 505-515.

Chen,A. (2001). A theoretical conceptualization for motivation research in physical education: An integrated perspective. Quest, 53, (1), 35-58.

Consejo Superior de Deportes (2015). Encuesta sobre los hábitos deportivos en España 2015. Madrid: Consejo Superior de Deportes.

Courel-Ibáñez, J., Cordero, J. C., Muñoz, D., Sánchez-Alcaraz, B. J., Grijota, F. J. \& Robles, M. C. (2018). Fitness benefits of padel practice in middle-aged adult women. Science and Sports. https://doi.org/10.1016/j.scispo.2018.01.011

Courel-Ibáñez, J., Sánchez-Alcaraz, B. J., \& Cañas, J. (2017). Game performance and length of rally in professional padel player. Journal of Human Kinetics, 55(1), 161169. https://doi.org/10.1515/hukin-2016-0045

Courel-Ibáñez, J., Sánchez-Alcaraz, B.J., García, S. \& Echegaray, M. (2017). Evolución del pádel en España en función del género y edad de los practicantes. Cultura, Ciencia y Deporte, 34(12) 39-46

Courel-Ibáñez, J., Sánchez-Alcaraz, B. J., \& Muñoz, D. (2019). Exploring Game Dynamics in Padel: Implications for Assessment and Training. Journal of Strength and Conditioning Research, 33(7), 1971-1977. https:// doi.org/10.1519/JSC.0000000000002126

Courel-Ibáñez, J., Sánchez-Alcaraz, B.J., Muñoz, D., Grijota, F. J., Chaparro, R., \& Díaz, J. (2018). Motivos de género para la práctica del pádel. Apunts Educación Física y Deportes, (133), 116-125. https://doi.org/10.5672/ apunts.2014-0983.es.(2018/3).133.08

Courel-Ibáñez, J., Sanchez-Alcaraz, B. J., \& Sánchez-Alcaraz, B. J. (2017). Efecto de las variables situacionales sobre los puntos en jugadores de pádel de élite. Apunts Edu- 
cación Física y Deportes, 121(1), 72-78. https://doi.org/ http://dx.doi.org/10.5672/apunts.20140983.es.\%282017/ $1 \% 29.127 .07$

Dosil, J. (2004). Motivación: motor del deporte. En J. Dosil (ed.), Psicología de la Actividad Física y del Deporte, Madrid: Mc Graw Hill.

Duda, J. L. y Nicholls, J. G. (1992). Dimensions of achievement motivation in schoolwork and sport. Journal of Educational Psychology, 84(3), 290-299.

Durand, M. (1988). El niño y el deporte. (2ª ed.). Barcelona: Ediciones Paidós.

García-Benítez, S., Courel-Ibáñez, J., Pérez-Bilbao, T., \& Felipe, J. L. (2018). Game responses during young padel match play: Age and sex comparisons. Journal of Strength and Conditioning Research, 32(4), 1144-1149. https:// doi.org/10.1519/JSC.0000000000001951

García-Puello, F., Herazo, Y., \& Tuesca, R. (2015). Factores sociodemográficos y motivacionales asociados a la actividad física en estudiantes universitarios. Revista Médica de Chile, 143, 1411-1418.

Gill, D. L., Gross, J. \& Huddleston, S. (1983). Participation motivation in youth sports. International Journal of Sport Psychology, 14, 1-14.

González, G., Tabernero, B. \& Márquez, S. (2000). Análisis de las motivaciones para participar en fútbol y en tenis en la iniciación deportiva. Revista Motricidad, 6, 47-66.

Hernández, J. L., Velázquez, R. \& Aguado, R. (2010). La educación física a estudio: el profesorado, el alumnado y los procesos de enseñanza. Barcelona: Graó.

Lasaga, M. J. (2011). Estudios social y metodológico del pádel desde la percepción de técnicos y jugadores: una apuesta educativa. Tesis Doctoral. Universidad de Sevilla.

López, C. y Márquez, S. (2001). Motivación en jóvenes practicantes de lucha leonesa. Revista de Psicología del Deporte, 10(1), 9-22.

Lupo, C., Condello, G., Courel-Ibáñez, J., Gallo, C., Conte, D., \& Tessitore,A. (2018). Effect of gender and match outcome on professional padel competition. RICYDE: Revista Internacional de Ciencias Del Deporte, 14(51), 29-41. https:/ /doi.org/10.5232/ricyde2018.05103

Martínez, R., Molinero, O., Jímenez, R., Salguero, A., Tuero, C. \& Márquez, S. (2008). La motivación para la práctica en la iniciación al fútbol: influencia de la edad/categoría competitiva, el tiempo de entrenamiento y la relación con el entrenador. Revista Apunts, 93, 46-54.

Martínez, A., Chillón, P., Martín, M., López, I., Castillo, R., Zapatera, B. \& Tercedor, P. (2012). Motivos de práctica de actividad físico-deportiva en adolescentes españoles: estudio AVENA. Profesorado. Revista de currículum y formación del profesorado, 16(1), 391-398

Moreno, J.A., Cervelló, E. \& González, D. (2007). Analizando la motivación en el deporte: un estudio a través de la teoría de la autodeterminación. Apuntes de Psicología, 25(1), 35-51.

Moreno, J. A. \& Cervelló, E. (2010). Motivación en la actividad física y el deporte. Sevilla: Wanceulen Editorial.

Moreno-Murcia, J.A.; Sicilia, A.; Sáenz-López, P.; GonzálezCutre, D.; Almagro, B.J. \& Conde, C. (2014). Análisis motivacional comparativo en tres contextos de actividad física / Motivational analysis comparing three contexts of physical activity. Revista Internacional de Medicina y Ciencias de la Actividad Física y el Deporte vol. 14 (56) pp. 665-685

Muñoz, D., García, A., Pérez, F. J., Díaz, J., Bartolome, I., \& Muñoz, J. (2016). Influencia de la duración del set sobre variables temporales de juego en pádel. Apunts. Educación Física y Deportes, 123, 69-75.

Nuviala, A., Gómez, M., Grao, A., Granero,A., \& Nuviala, R. (2013). Perfiles motivacionales de usuarios de servicios deportivos públicos y privados. Universitas Psychologica, 12(2), 421-431.

Pavón, A. \& Moreno, J.A. (2008). Actitud de los universitarios ante la práctica físico-deportiva: diferencias por géneros. Revista de Psicología del Deporte, 7(1), 7-23.

Pérez, S., Sánchez, J. \& Urchaga, J. D. (2015). Los motivos para la participación en fútbol: estudio por categorías y nivel de competición de los jugadores. Cultura, Ciencia y Deporte, 10(30), 187-198.

Rodríguez-Cayetano, A., Sánchez, A., De Mena, J. M., Fuentes, J. M., Castaño, R. \& Pérez, S. (2017). Pre-competitive anxiety in $\mathrm{U} 12, \mathrm{U} 14$ and $\mathrm{U} 16$ paddle tennis players. Revista de Psicología del Deporte, 26(3), 51-56.

Ruiz, R. \& Lorenzo, O. (2008). Características psicológicas en los jugadores de pádel de alto rendimiento. Revista iberoamericana de psicología del ejercicio y el deporte, 3(2), 183-199.

Ruiz-Juan, F., Gómez, M., Pappous, A., Alacid, F. \& Flores, G. (2010). Dispositional goal orientation, beliefs about the causes of success and intrinsic satisfaction in young elite players. Journal of Human Kinetics, 26, 123-136.

Sánchez-Alcaraz, B. J. (2013). Historia del Pádel. Materiales para la Historia del Pádel, 11, 57-60.

Sánchez-Alcaraz, B. J., Courel-Ibáñez, J., \& Cañas, J. (2018). Temporal structure, court movements and game actions in padel: A systematic review. Retos, 33(33), 308-312.

Sánchez-Alcaraz, Muñoz, D., Courel-Ibañez, J., Grijota, F. J., Chaparro, R., \& Díaz, J. (2018). Motivos de la práctica del pádel en relación a la edad, el nivel de juego y el género. SPORT TK-Revista EuroAmericana de Ciencias del Deporte, 7(1), 57. https://doi.org/10.6018/321881

Sañudo, B., Hoyo, M. D. \& Carrasco, L. (2008). Demandas fisiológicas y características estructurales de la competición en pádel masculino. Apunts. Educación física y deportes, 94, 23-28.

Zarauz, A. \& Ruiz-Juan, F. (2014). Análisis de la motivación en el atletismo: un estudio con veteranos. Universitas Psychologica, 13(2), 501-516.

Zubiaur, M. \& Del Riego, M. (2015). Motivaciones e intereses de practicantes de BMX adolescentes: un estudio piloto. Retos, 27, 109-113.

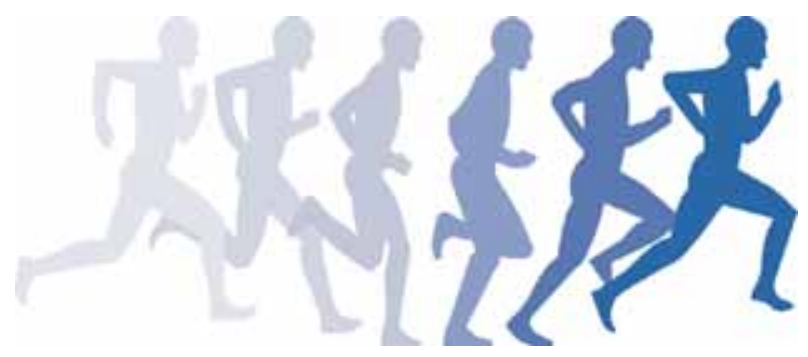

